\title{
Interobserver variability in intraductal papillary mucinous neoplasm subtypes and application of their mucin immunoprofiles
}

\author{
Heewon A Kwak ${ }^{1}$, Xiuli Liu ${ }^{2}$, Daniela S Allende ${ }^{3}$, Rish K Pai ${ }^{4}$, John Hart ${ }^{1}$ and \\ Shu-Yuan Xiao ${ }^{1}$
}

${ }^{1}$ Department of Pathology, University of Chicago, Chicago, IL, USA; ${ }^{2}$ Department of Pathology, University of Florida, Gainesville, FL, USA; ${ }^{3}$ Department of Pathology, Cleveland Clinic Foundation, Cleveland, OH, USA and ${ }^{4}$ Department of Pathology, Mayo Clinic, Scottsdale, AZ, USA

\begin{abstract}
Intraductal papillary mucinous neoplasm is considered a precursor lesion to pancreatic adenocarcinoma. These are further classified into four histologic subtypes: gastric, intestinal, pancreatobiliary, and oncocytic. The first aim of this study was to assess the interobserver variability among five gastrointestinal pathologists in diagnosing intraductal papillary mucinous neoplasm subtypes by morphology alone. The second aim of the study was to compare intraductal papillary mucinous neoplasm subtypes, which received consensus diagnoses ( $\geq 80 \%$ agreement) with their respective mucin immunoprofiles (MUC1, MUC2, MUC5AC, MUC6, and CDX2). A consensus histologic subtype was reached in $58 \%$ of cases (29/50) among the five gastrointestinal pathologists. Overall there was moderate agreement $(\kappa=0.41, P<0.01)$ in subtyping intraductal papillary mucinous neoplasms without the use of immunohistochemistry. The histologic subtype with the best interobserver agreement was intestinal type $(\kappa=0.56, P<0.01)$ followed by pancreatobiliary, gastric, mixed, and oncocytic types $(\kappa=0.43$, $P<0.01 ; \kappa=0.38, P<0.01 ; \kappa=0.17, P<0.01 ; \kappa=0.08, P<0.04$, respectively). Both kappa values for mixed and oncocytic subtypes were likely artificially low due to the underrepresentation of these subtypes in this study and not a true indication of poor interobserver agreement. Following an intradepartmental consensus meeting between two gastrointestinal pathologists, $68 \%$ of cases $(34 / 50)$ received a consensus intraductal papillary mucinous neoplasm subtype. Sixty-nine percent of cases (11/16) that did not receive a consensus intraductal papillary mucinous neoplasm subtype could be classified based on their respective immunoprofiles. Standardizing the use of immunohistochemistry with a mucin immunopanel (MUC1, MUC2, MUC5AC, and MUC6) may improve the agreement of diagnosing intraductal papillary mucinous neoplasm histologic subtypes. Modern Pathology (2016) 29, 977-984; doi:10.1038/modpathol.2016.93; published online 13 May 2016
\end{abstract}

Intraductal papillary mucinous neoplasm (IPMN) was coined in 1994 and is defined as a preinvasive pancreatic neoplasm arising in pancreatic ducts that forms a grossly visible mass. ${ }^{1}$ A consensus meeting in 2003 further classified IPMN into four histologic subtypes: gastric, intestinal, pancreatobiliary, and oncocytic based on morphology and immunohistochemistry. ${ }^{2}$ MUC1, MUC2, and MUC5AC immunohistochemical stains were used in the initial classification. Additional studies observed MUC6 and CDX2 as useful gastric and intestinal markers in IPMNs, respectively. ${ }^{3,4}$

Correspondence: Dr S-Y Xiao, MD, Department of Pathology, University of Chicago Medicine, 5841 S. Maryland Ave., MC6101, Chicago, IL 60637, USA

E-mail: syxiao@uchicago.edu

Received 17 February 2016; revised 15 April 2016; accepted 16 April 2016; published online 13 May 2016
Following categorization into histologic subtypes, multiple clinicopathological correlation studies were performed. Gastric subtype is commonly seen in branch duct IPMNs whereas intestinal and pancreatobiliary subtypes usually involve the main pancreatic duct. Gastric and pancreatobiliary subtypes are more often associated with invasive tubular adenocarcinoma versus intestinal subtype, which is frequently associated with invasive colloid carcinoma. $^{5-8}$ Furthermore, studies have reported conflicting evidence on whether the histologic IPMN subtype has prognostic significance..$^{8-12}$

The Verona consensus meeting in 2013 recently led to the publication of guidelines for pathologic reporting of IPMN with the recommendation that pathologists provide a histologic subtype. ${ }^{13}$ Although most previous studies used morphology and immunohistochemistry to designate the IPMN subtype, this is not necessarily implemented as 
standard practice. Our study analyzes interobserver agreement between specialized gastrointestinal pathologists to subtype IPMN using morphology alone. In addition, we compare their consensus morphologic diagnoses with the immunohistochemical profiles for each case.

\section{Materials and methods}

\section{Case Selection}

The study included 50 cases of IPMN during 20002013 from the surgical pathology archives of the University of Chicago (27 cases) and Cleveland Clinic (23 cases). Histological slides of the cases were reviewed by pathologist (SX) or (XL) with one representative hematoxylin and eosin stained slide selected from each case. The criterion for the representative slide was that there would be substantial lesional tissue to make a histologic subtype diagnosis without discriminating between the presence of one or more subtypes.

\section{Interobserver Agreement}

Five pathologists with specialized training in gastrointestinal pathology were chosen to individually review the cases and subtype each IPMN as gastric, intestinal, pancreatobiliary, oncocytic, or mixed. Mixed subtype was designated as having more than one subtype, each comprising $\geq 25 \%$ of the lesion. Additional parameters recorded were the grade of dysplasia (low, intermediate, or high) and the presence or absence of invasion. All the data received were generated based on hematoxylin and eosin stained slides without the use of special or immunohistochemical stains.

A consensus diagnosis for each category was defined as having four of five pathologists in agreement with one another, or $\geq 80 \%$.

Cases that did not meet the $80 \%$ agreement for IPMN subtyping were revisited by two pathologists at an intradepartmental consensus meeting. The main reason of this consensus meeting was to render a 'final' histotype designation for subsequent immunophenotypic study, when possible.

IPMN subtypes were designated for each case when there was $\geq 80 \%$ interobserver agreement following the intradepartmental consensus meeting. These cases were subsequently correlated with the immunohistochemical panel performed. The cases with $<80 \%$ interobserver agreement were categorized into IPMN subtypes using previously published immunohistochemical data on IPMN classification, when possible..$^{2,4}$

\section{Immunohistochemical Studies}

All 50 cases of IPMN had immunohistochemical staining performed on $5-\mu \mathrm{m}$ tissue sections from the same formalin-fixed, paraffin-embedded tissue block, which was selected for initial hematoxylin and eosin stained slide examination. MUC1 (MA695; NovoCastra, UK; 1:400), MUC2 (CCP58; NovoCastra; 1:25), MUC5AC (CLH2; NovoCastra; 1:25), MUC6 (CLH5; NovoCastra; 1:25), and CDX2 (CDX2-88; Biogenex, Fremont, CA, USA; 1:100) immunohistochemical staining was performed on all cases. The immunostains had appropriate controls and were reviewed by one pathologist (SX). The immunohistochemistry was quantitatively scored based on percent immunoreactivity in the lesional cells. A positive result was associated with $\geq 10 \%$ positive staining and a negative result associated with $<10 \%$ positive staining.

\section{Statistical Analysis}

The interobserver agreements were calculated using Fleiss' kappa statistic $(\kappa)$. A $\kappa$-value ranging from 0.00 to 0.20 is slight agreement, $0.21-0.40$ is fair agreement, $0.41-0.60$ is moderate agreement, $0.61-0.80$ is substantial agreement, and $0.81-1.00$ is almost perfect agreement. ${ }^{14}$

\section{Results}

\section{Agreement of IPMN Histological Subtypes}

The interobserver variability for IPMN subtypes is summarized in Table 1 . There was moderate agreement $(\kappa=0.41, P<0.01)$ among the five gastrointestinal pathologists, overall. Intestinal subtype had the best agreement $(\kappa=0.56, P<0.01)$, while oncocytic subtype had the poorest agreement $(\kappa=0.08$, $P<0.04)$. Figure 1 represents cases of IPMN subtypes that received a consensus subtype followed by cases that lacked a consensus subtype and were classified by pathologists as either one of two specific subtypes.

\section{Agreement of Grade of Dysplasia and Invasion}

Table 2 shows results of interobserver variability in determining the presence or absence of dysplasia

Table 1 Kappa-values for interobserver reproducibility of intraductal papillary mucinous neoplasm subtyping

\begin{tabular}{lcc}
\hline & $\kappa$-Value $(95 \%$ confidence \\
interval) & P-value \\
\hline Subtypes & & \\
Gastric & $0.38(0.30-0.47)$ & $<0.00001$ \\
Intestinal & $0.56(0.47-0.64)$ & $<0.00001$ \\
Pancreatobiliary & $0.43(0.34-0.51)$ & $<0.00001$ \\
Oncocytic & $0.08(0-0.17)$ & $<0.04000$ \\
Mixed & $0.17(0.08-0.26)$ & $<0.00001$ \\
Overall $\kappa$-value & $0.41(0.35-0.46)$ & $<0.00001$ \\
\hline
\end{tabular}



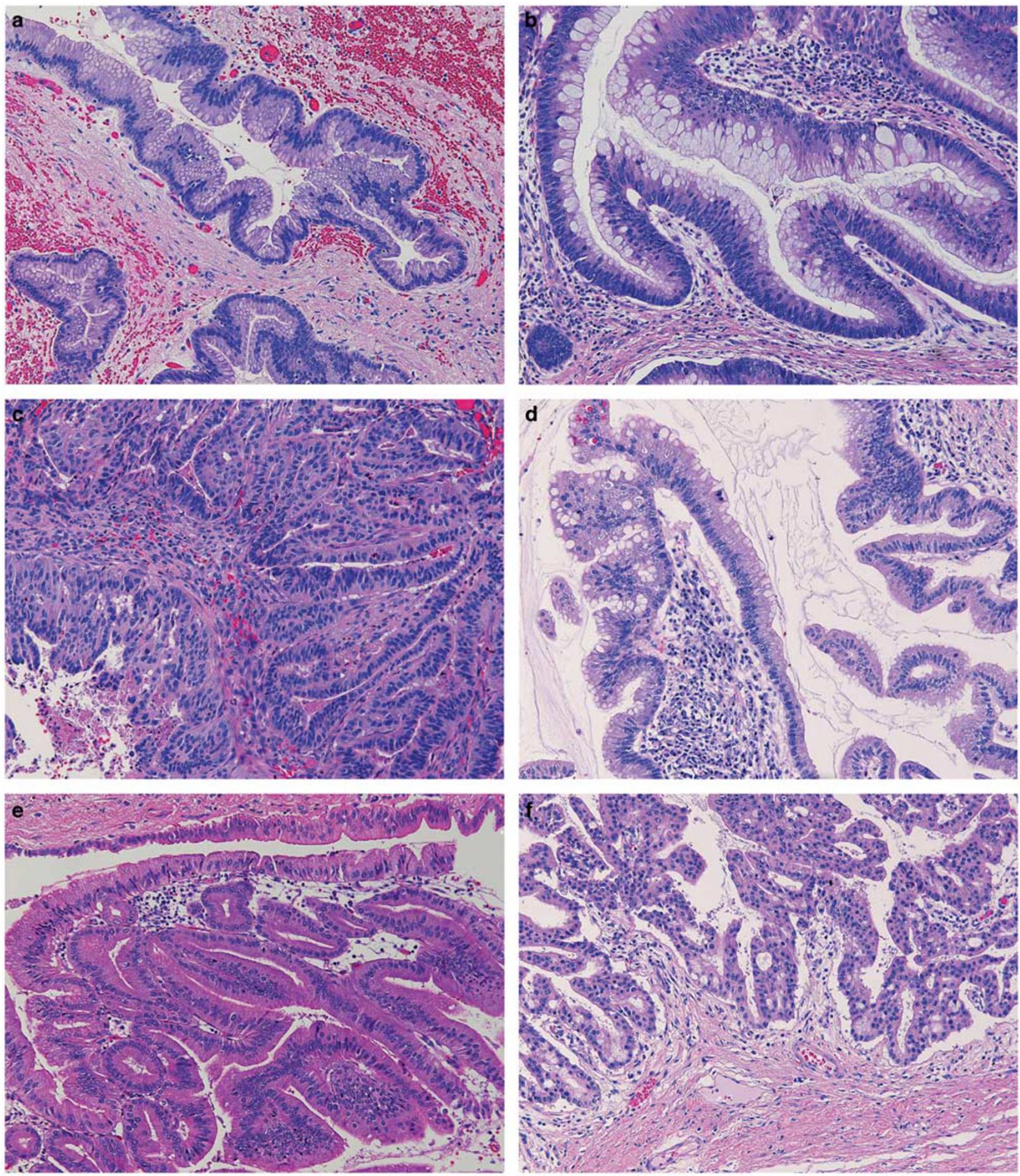

Figure 1 Representative hematoxylin and eosin stained slide images of the different morphologic subtypes of intraductal papillary mucinous neoplasm seen in our case series. Consensus diagnoses were achieved for gastric subtype (a), intestinal subtype (b), and pancreatobiliary subtype (c) in these cases. No consensus between gastric or intestinal subtype (d), no consensus between gastric or pancreatobiliary subtype (e), and no consensus for pancreatobiliary or oncocytic subtype (f) were achieved in these cases.

and invasion. Identifying low- and high-grade dysplasia had moderate agreement $(\kappa=0.45, P<0.01$ and $\kappa=0.60, P<0.01$, respectively) whereas intermediate dysplasia had slight agreement (0.18,
$P<0.01)$. Overall, there was moderate $(\kappa=0.41$, $P<0.01)$ and substantial $(\kappa=0.72, P<0.01)$ agreement between the pathologists grading dysplasia and recognizing invasion, respectively. 
Table 2 Kappa-values for interobserver reproducibility of grading dysplasia and identifying invasion in intraductal papillary mucinous neoplasm

\begin{tabular}{|c|c|c|c|}
\hline & $\begin{array}{c}\text { No. of cases classified with } \geq 80 \% \text { agreement } \\
\text { (\% of consensus cases) }\end{array}$ & $\begin{array}{l}\text { к-Value (95\% confidence } \\
\text { interval) }\end{array}$ & $\mathrm{P}$-value \\
\hline \multicolumn{4}{|l|}{ Dysplasia } \\
\hline Low & 11 & $0.45(0.36-0.54)$ & $<0.00001$ \\
\hline Intermediate & 6 & $0.18(0.10-0.27)$ & 0.00002 \\
\hline High & 14 & $0.60(0.51-0.69)$ & $<0.00001$ \\
\hline Overall $\kappa$-value & & $0.41(0.35-0.47)$ & $<0.00001$ \\
\hline $\begin{array}{l}\text { Total no. of cases with } \geq 80 \% \\
\text { agreement (\% cases) }\end{array}$ & $31(62 \%)$ & & \\
\hline Presence or absence of invasion & $46(92 \%)$ & $0.72(0.63-0.81)$ & $<0.00001$ \\
\hline
\end{tabular}

\section{Correlation Between Histological Subtyping and Grade of Dysplasia}

Cases designated as having low-grade dysplasia consisted of $72.7 \%$ gastric, $0.0 \%$ intestinal, $0.0 \%$ pancreatobiliary subtypes, respectively and $27.2 \%$ without a consensus subtype. Intermediate grade dysplasia cases comprised $16.7 \%$ gastric, $16.7 \%$ intestinal, $0.0 \%$ pancreatobiliary, and $66.7 \%$ no consensus subtypes, respectively. Finally, highgrade cases were gastric, $14.3 \%$ intestinal, $21.4 \%$ pancreatobiliary, and $64.3 \%$ no consensus subtypes, respectively.

Recent Baltimore Consensus Meeting recommendations suggest collapsing the three-tiered system for grading dysplasia in pancreatic precursor lesions into two by combining low and intermediate grades and relabeling them all as low grade. ${ }^{15}$ After applying the two-tiered system, low-grade cases consisted of $56.7 \%$ gastric, $10.0 \%$ intestinal, $0.0 \%$ pancreatobiliary, and $33.3 \%$ no consensus subtypes, respectively, whereas high-grade dysplasia comprised $0.0 \%$ gastric, $14.3 \%$ intestinal, $21.4 \%$ pancreatobiliary, and $64.3 \%$ no consensus, subtypes, respectively.

\section{Correlation Between Histological Subtyping and Immunohistochemistry}

Table 3 summarizes the percent of cases that received a consensus diagnosis based on hematoxylin and eosin stained slides before and after an intradepartmental consensus meeting (58 and 68\%, respectively). Thirty-four cases were classified as a specific subtype based on achieving a consensus diagnosis. The immunohistochemical profiles for these IPMN subtypes are shown in Table 4. MUC5AC followed by MUC6 were markers most commonly seen in gastric subtypes. MUC2 and CDX2 were mostly seen only in intestinal subtypes; however, MUC5AC was also seen in two-thirds of cases $^{6}$ classified as intestinal subtype. Pancreatobiliary subtype did not have a consistent staining pattern, but was the only subtype with positive immunoreactivity for MUC1. In Figure 2, examples of IPMN
Table 3 Submitted intraductal papillary mucinous neoplasm subtypes by morphology

\begin{tabular}{lcc}
\hline & $\begin{array}{c}\text { No. of cases classified } \\
\text { with } \geq 80 \% \\
\text { agreement } \% \text { of } \\
\text { consensus cases) }\end{array}$ & $\begin{array}{c}\text { No. of cases } \\
\text { following } \\
\text { consensus } \\
\text { meeting (\%) }\end{array}$ \\
\hline $\begin{array}{l}\text { Subtypes } \\
\text { Gastric }\end{array}$ & 18 & \\
Intestinal & 7 & 20 \\
Pancreatobiliary & 4 & 9 \\
Oncocytic & 0 & 5 \\
Mixed & $0^{\mathrm{a}}$ & 0 \\
Total no. of cases & $29(58 \%)$ & $0^{\mathrm{a}}$ \\
with $\geq 80 \%$ agreement & & \\
$(\%$ cases) & &
\end{tabular}

${ }^{a}$ Although cases were subtyped as mixed in certain cases, a consensus was not reached on which subtypes were present within the mix.

Table 4 Correlating intraductal papillary mucinous neoplasm histologic subtypes with immunohistochemistry

\begin{tabular}{lccccc}
\hline & MUC1 & MUC2 & MUC5AC & MUC6 & CDX2 \\
\hline Gastric & 0 & 1 & 20 & 13 & 0 \\
Intestinal & 0 & 9 & 6 & 0 & 8 \\
Pancreatobiliary & 3 & 1 & 3 & 1 & 1 \\
\hline
\end{tabular}

subtypes and their respective immunoprofiles are presented.

Additionally, Figure 2 demonstrates a case without an assigned consensus IPMN subtype by morphology that can instead be classified based on immunohistochemistry. Overall, 11 of the 16 cases without a consensus subtype had a specific immunoprofile as seen in Table 5. Of the 11 cases, seven and four stained like gastric (MUC1-, MUC2 -, MUC5AC+, MUC6+, and CDX2-) and intestinal (MUC1 -, MUC2+, MUC5AC \pm , MUC6 - , and CDX2 \pm ) subtypes, respectively. The remaining 

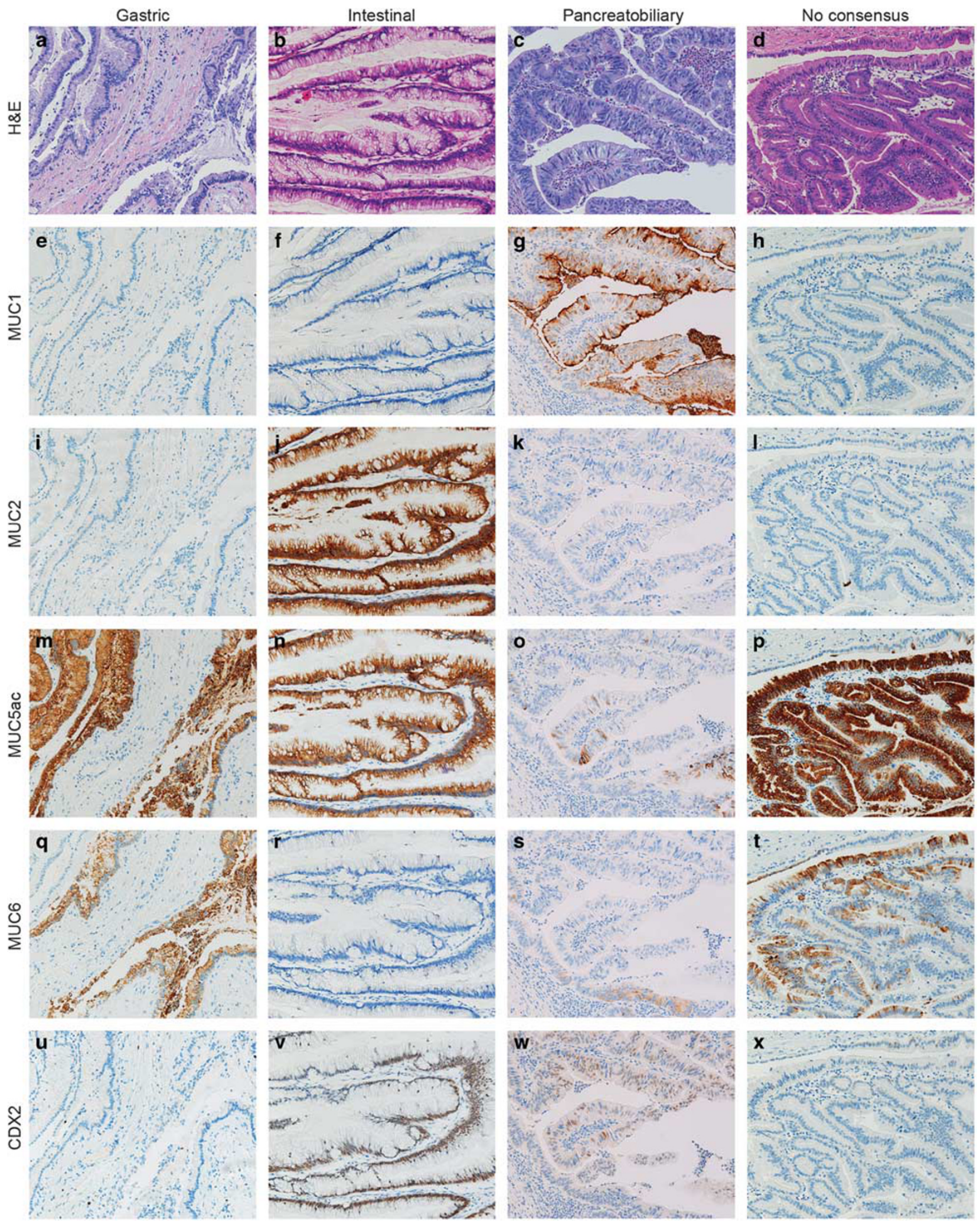

Figure 2 Hematoxylin and eosin stained slide images of the different morphologic subtypes of intraductal papillary mucinous neoplasm with corresponding immunohistochemical images. Representative images of cases with a consensus diagnosis of gastric subtype (a), intestinal subtype (b), and pancreatobiliary subtype (c). No consensus between gastric or pancreatobiliary subtypes was reached in this case (d). MUC5ac and MUC6 were positive (m, q), while MUC1, MUC2, and CDX2 were negative (e, i, u) in this case with gastric subtype. MUC2, MUC5ac, and CDX2 were positive ( $\mathbf{j}, \mathbf{n}, \mathbf{v})$, while MUC1 and MUC6 were negative (f, $\mathbf{r})$ in this case with intestinal subtype. MUC1 and CDX2 were positive (g, w), while MUC2, MUC5ac, and MUC6 were negative (k,o,s) in this case with pancreatobiliary subtype. The case without a consensus diagnosis was MUC5ac and MUC6 positive (p, t), while MUC1, MUC2, and CDX2 negative (h, l, x). 
Table 5 Correlating cases with no consensus on histologic subtypes with immunohistochemistry

\begin{tabular}{|c|c|c|c|c|c|c|}
\hline Case \# & MUC1 & MUC2 & MUC5AC & MUC6 & $C D X 2$ & $\begin{array}{l}\text { Subtype } \\
\text { following } \\
\text { immuno- } \\
\text { histochemistry }\end{array}$ \\
\hline 3 & - & - & - & - & - & Inconclusive \\
\hline 8 & - & - & + & + & - & Gastric \\
\hline 12 & - & - & + & - & - & Inconclusive \\
\hline 20 & - & + & + & + & + & Mixed \\
\hline 23 & - & + & + & - & + & Intestinal \\
\hline 27 & - & - & + & + & - & Gastric \\
\hline 28 & - & + & + & - & - & Intestinal \\
\hline 29 & - & - & + & + & - & Gastric \\
\hline 30 & - & - & + & + & + & Mixed \\
\hline 32 & - & + & - & - & + & Intestinal \\
\hline 33 & - & - & + & - & - & Inconclusive \\
\hline 34 & - & + & + & - & + & Intestinal \\
\hline 36 & - & - & + & + & - & Gastric \\
\hline 38 & - & - & + & + & - & Gastric \\
\hline 40 & - & - & + & + & - & Gastric \\
\hline 48 & - & - & + & + & - & Gastric \\
\hline
\end{tabular}

five cases had either no staining, (1) MUC5AC+ only (2) or immunoprofiles supportive of mixed subtypes (2).

\section{Discussion}

In this study, we show moderate agreement $(\kappa=0.41$, $P<0.01)$ among five gastrointestinal pathologists in classifying IPMN subtypes using morphology alone with $58 \%$ of cases $(29 / 50)$ receiving a consensus IPMN subtype. Additionally, 69\% of IPMN cases without a consensus subtype (11/16) could be classified based on their immunoprofiles. A previous interobserver study grading Barrett's dysplasia among gastrointestinal pathologists by Downs-Kelly et $a{ }^{16}$ demonstrated fair overall agreement $(\kappa=0.30)$, while another study by Kaye et $a l^{17}$ had improved overall interobserver agreement in grading Barret's dysplasia following the use of p53 immunohistochemistry ( $\kappa=0.42$ to $\kappa=0.48$ after the use of immunohistochemistry). These studies illustrate that moderate agreement in interobserver studies is not necessarily easy to achieve and that judicious use of immunohistochemistry can help with interobserver agreement.

As the largest interobserver study of IPMN in the literature, comprising five pathologists specialized in gastrointestinal pathology, this study demonstrates the difficulty of subtyping IPMN and the ability of immunohistochemistry to help subtype in cases with ambiguous morphology. Currently in our daily practice, we traditionally rely on morphology alone to determine the IPMN subtype in our resection specimens. However, most published clinicopathological correlation studies on IPMN use a combined method of morphology and immunohistochemistry to histologically subtype IPMN. Thus, in accordance to the recent guidelines that recommend pathologists provide a histologic subtype when reporting IPMN, we suggest the use of immunohistochemistry in cases when a definitive subtype cannot be assigned with morphology alone.

A recent publication by Schaberg et $a l^{18}$ reported limited value in the use of immunohistochemistry in subtyping IPMN using the WHO classification for IPMN immunophenotypes. In their study immunohistochemistry was only performed on equivocal cases and only two of their nine ambiguous cases were further classified using immunohistochemistry. In the current study there are cases where a consensus diagnosis of an IPMN subtype was achieved using morphology on hematoxylin and eosin stained slides alone with corresponding immunohistochemistry demonstrating an overlap of mucin markers or the lack of specific MUC immunohistochemical staining. Our findings correlated with previous studies where MUC2 and CDX2 were seen in nonintestinal subtypes and most but not all intestinal subtypes had immunoreactivity with CDX2. ${ }^{3,4}$ When CDX2 was classified as positive (defined as $\geq 10 \%$ immunoreactivity) in the intestinal subtypes though, all cases showed that $\geq 50 \%$ of the lesional cells were positive. In addition, a lack of staining may also be seen in areas with high-grade dysplasia. Recognizing the limitations of immunohistochemistry, we recommend using it in conjunction with morphology, deferring to morphology to aid in differentiating discrepant immunohistochemical staining from the presence of more than one subtype.

Our study confirms the utility of MUC stains, especially MUC2 in identifying IPMN as intestinal subtype. In addition, MUC6 positivity in our study differentiates gastric from intestinal and pancreatobiliary subtypes, which can also be positive for MUC5AC. Similar to Basturk et al, MUC6 is positive in flat or cystic spaces with pyloric-like histology seen in our cases identified as gastric subtype. In cases identified as pancreatobiliary subtype however, only one of our cases had immunoreactivity with MUC6, unlike their study, which had weak but consistent expression of MUC6 in their pancreatobiliary IPMNs. This however may reflect our low number of pancreatobiliary cases. ${ }^{19}$ Previous studies have distinguished MUC1 as an aggressive marker in pancreatic neoplasms, positive in areas of invasive carcinoma and IPMN with high-grade dysplasia. ${ }^{20-22}$ Though limited in the number of cases, the pancreatobiliary subtypes of IPMN in our study were the only ones with positivity for MUC1. We propose the ideal immunopanel in classifying IPMN subtypes to consist of MUC1, MUC2, MUC5AC, and MUC6.

Overall, sample size and unequal representation of all the subtypes were limitations in this study. This limitation is demonstrated by the poor interobserver agreement seen in the mixed and oncocytic subtypes, which we would expect to have better agreement in the setting of more cases. A study by Reid et $a l^{23}$ demonstrated the ability to accurately provide a cytopathologic diagnosis of intraductal onococytic papillary neoplasms using helpful cytomorphologic 
features and Basturk et $a l^{24}$ demonstrated distinct molecular characteristics in this subtype. This is significant given evidence that the oncocytic subtype carries a favorable long-term prognosis. ${ }^{25,26}$ Nevertheless, the cases in our study were representative of the IPMN specimens received at both institutions. Previous studies also demonstrate the greater prevalence of gastric and intestinal IPMN subtypes. , $^{7,8,27}$ The mixed subtype may have been difficult to assess due to the limited examination of the specimen by all the gastrointestinal pathologists, which is reflected in the poor interobserver agreement for this subtype. Nonetheless, the entirety of each case was seen by a gastrointestinal pathologist at his or her respective institution and the selected slide from each case should be considered representative of the overall lesion.

Although the clinicopathological significance of subtyping IPMN in a resection specimen is equivocal, the importance of identifying an invasive component for prognosis is undisputed. There was substantial agreement $(\kappa=0.72, P<0.001)$ in this study for diagnosing invasion. While this received the highest $\kappa$-value, there was not complete concordance among the pathologists in all the cases. Hence, comprehensive sampling of the specimen is essential to ensure invasion is not missed since the associated presence of invasive adenocarcinoma affects patient prognosis and management.

Interestingly, classification of IPMN subtypes has been attempted preoperatively through immunohistochemical studies of pancreatic juice cytology by Hara et al. ${ }^{28}$ Histological IPMN subtype diagnosed with pancreatic juice cytology was compared with the subtype diagnosis made at resection. With the use of MUC1, MUC2, and MUC5AC immunostains, the histological IPMN subtype was correctly diagnosed in $89 \%$ of cases $(32 / 36)$ with a sensitivity and specificity of 86 and $100 \%$, respectively. In order to stratify high- and low-risk patients with IPMN, Maker et $a 1^{29}$ and Yokoyama et $a l^{30}$ looked at various mucin expression profiles in pancreatic cyst fluid and pancreatic juice through enzyme immunoassay and DNA methylation analysis, respectively. These studies use mucin expression profiles to help identify IPMN subtypes and highlight a potential use of IPMN subtyping in helping guide the surgeon when determining surgical management.

\section{Disclosure/conflict of interest}

The authors declare no conflict of interest.

\section{References}

1 Sessa F, Solcia E, Capella C et al. Intraductal papillarymucinous tumours represent a distinct group of pancreatic neoplasms: an investigation of tumour cell differentiation and K-ras, p53 and c-erbB-2 abnormalities in 26 patients. Virchows Arch 1994;425:357-367.
2 Furukawa T, Kloppel G, Volkan Adsay N et al. Classification of types of intraductal papillary-mucinous neoplasm of the pancreas: a consensus study. Virchows Arch 2005;447:794-799.

3 Adsay NV, Merati K, Basturk O et al. Pathologically and biologically distinct types of epithelium in intraductal papillary mucinous neoplasms: delineation of an "intestinal" pathway of carcinogenesis in the pancreas. Am J Surg Pathol 2004;28:839-848.

4 Kobayashi M, Fujinaga Y, Ota H. Reappraisal of the immunophenotype of pancreatic intraductal papillary mucinous neoplasms (IPMNs)-gastric pyloric and small intestinal immunophenotype expression in gastric and intestinal type IPMNs. Acta Histochem Cytochem 2014;47:45-57.

5 Yamada S, Fujii T, Shimoyama Y et al. Clinical implication of morphological subtypes in management of intraductal papillary mucinous neoplasm. Ann Surg Oncol 2014;21:2444-2452.

6 Mino-Kenudson M, Fernandez-del Castillo C, Baba Y et al. Prognosis of invasive intraductal papillary mucinous neoplasm depends on histological and precursor epithelial subtypes. Gut 2011;60:1712-1720.

7 Furukawa T, Hatori T, Fujita I et al. Prognostic relevance of morphological types of intraductal papillary mucinous neoplasms of the pancreas. Gut 2011;60: 509-516.

8 Ishida M, Egawa S, Aoki T et al. Characteristic clinicopathological features of the types of intraductal papillary-mucinous neoplasms of the pancreas. Pancreas 2007;35:348-352.

9 Kim J, Jang KT, Mo Park S et al. Prognostic relevance of pathologic subtypes and minimal invasion in intraductal papillary mucinous neoplasms of the pancreas. Tumour Biol 2011;32:535-542.

10 Kang MJ, Lee KB, Jang JY et al. Evaluation of clinical meaning of histological subtypes of intraductal papillary mucinous neoplasm of the pancreas. Pancreas 2013;42:959-966.

11 Koh YX, Zheng HL, Chok AY et al. Systematic review and meta-analysis of the spectrum and outcomes of different histologic subtypes of noninvasive and invasive intraductal papillary mucinous neoplasms. Surgery 2015;157:496-509.

12 Distler M, Kersting S, Niedergethmann M et al. Pathohistological subtype predicts survival in patients with intraductal papillary mucinous neoplasm (IPMN) of the pancreas. Ann Surg 2013;258:324-330.

13 Adsay V, Mino-Kenudson M, Furukawa T et al. Pathologic evaluation and reporting of intraductal papillary mucinous neoplasms of the pancreas and other tumoral intraepithelial neoplasms of pancreatobiliary tract: recommendations of Verona consensus meeting. Ann Surg 2016;263:162-177.

14 Landis JR, Koch GG. The measurement of observer agreement for categorical data. Biometrics 1977;33: 159-174.

15 Basturk O, Hong SM, Wood LD et al. A revised classification system and recommendations from the Baltimore consensus meeting for neoplastic precursor lesions in the pancreas. Am J Surg Pathol 2015;39: 1730-1741.

16 Downs-Kelly E, Mendelin JE, Bennett AE et al. Poor interobserver agreement in the distinction of highgrade dysplasia and adenocarcinoma in pretreatment Barrett's esophagus biopsies. Am J Gastroenterol 2008; 103:2333-2340; quiz 41. 
17 Kaye PV, Haider SA, Ilyas M et al. Barrett's dysplasia and the Vienna classification: reproducibility, prediction of progression and impact of consensus reporting and p53 immunohistochemistry. Histopathology 2009; 54:699-712.

18 Schaberg KB, DiMaio MA, Longacre TA. Intraductal papillary mucinous neoplasms often contain epithelium from multiple subtypes and/or are unclassifiable. Am J Surg Pathol 2016;40:44-50.

19 Basturk O, Khayyata S, Klimstra DS et al. Preferential expression of MUC6 in oncocytic and pancreatobiliary types of intraductal papillary neoplasms highlights a pyloropancreatic pathway, distinct from the intestinal pathway, in pancreatic carcinogenesis. Am J Surg Pathol 2010;34:364-370.

20 Yonezawa S, Nakamura A, Horinouchi $\mathrm{M}$ et al. The expression of several types of mucin is related to the biological behavior of pancreatic neoplasms. J Hepatobiliary Pancreat Surg 2002;9:328-341.

21 Nakamura A, Horinouchi M, Goto M et al. New classification of pancreatic intraductal papillary-mucinous tumour by mucin expression: its relationship with potential for malignancy. J Pathol 2002;197:201-210.

22 Yonezawa S, Taira M, Osako M et al. MUC-1 mucin expression in invasive areas of intraductal papillary mucinous tumors of the pancreas. Pathol Int 1998;48: 319-322.

23 Reid MD, Stallworth CR, Lewis MM et al. Cytopathologic diagnosis of oncocytic type intraductal papillary mucinous neoplasm: criteria and clinical implications of accurate diagnosis. Cancer Cytopathol 2016;124: 122-134.

24 Basturk O, Bhanot U, Berger M et al. Intraductal oncocytic papillary neoplasm of the pancreas have distinct molecular characteristics than intraductal papillary mucinous neoplasm (abstract 1758). Mod Pathol 2015;28:439.

25 Marchegiani G, Mino-Kenudson M, Ferrone CR et al. Oncocytic-type intraductal papillary mucinous neoplasms: a unique malignant pancreatic tumor with good long-term prognosis. J Am Coll Surg 2015;220:839-844.

26 Askan G, Klimstra DS, Adsay V et al. "Oncocytic-type" of intraductal papillary mucinous neoplasm (IPMN): an analysis of 25 cases (abstract 1740). Mod Pathol 2016;28:439.

27 Takasu N, Kimura W, Moriya $\mathrm{T}$ et al. Intraductal papillary mucinous neoplasms of the gastric and intestinal types may have less malignant potential than the pancreatobiliary type. Pancreas 2010;39:604-610.

28 Hara T, Ikebe D, Odaka A et al. Preoperative histological subtype classification of intraductal papillary mucinous neoplasms (IPMN) by pancreatic juice cytology with MUC stain. Ann Surg 2013;257:1103-1111.

29 Maker AV, Katabi N, Gonen M et al. Pancreatic cyst fluid and serum mucin levels predict dysplasia in intraductal papillary mucinous neoplasms of the pancreas. Ann Surg Oncol 2011;18:199-206.

30 Yokoyama S, Kitamoto S, Higashi M et al. Diagnosis of pancreatic neoplasms using a novel method of DNA methylation analysis of mucin expression in pancreatic juice. PLoS One 2014;9:e93760. 